\title{
Olivier Georges, Pierre-Marie Gerlier, le cardinal militant 1880-1965
}

Paris, Desclée de Brouwer, 2014, 474 p.

\section{Frédéric Le Moigne}

\section{(Q) OpenEdition}

\section{Journals}

Édition électronique

URL : http://journals.openedition.org/assr/26422

DOI : $10.4000 /$ assr.26422

ISSN : $1777-5825$

Éditeur

Éditions de l'EHESS

Édition imprimée

Date de publication : 31 décembre 2014

Pagination : 192

ISBN : 978-2-7132-2467-6

ISSN : 0335-5985

\section{Référence électronique}

Frédéric Le Moigne, "Olivier Georges, Pierre-Marie Gerlier, le cardinal militant 1880-1965 », Archives de sciences sociales des religions [En ligne], 168 | 2014, mis en ligne le 17 avril 2015, consulté le 22 septembre 2020. URL : http://journals.openedition.org/assr/26422 ; DOI : https://doi.org/10.4000/ assr.26422

Ce document a été généré automatiquement le 22 septembre 2020

(c) Archives de sciences sociales des religions 
Olivier Georges, Pierre-Marie Gerlier, le cardinal militant 1880-1965

Paris, Desclée de Brouwer, 2014, 474 p.

Frédéric Le Moigne

\section{RÉFÉRENCE}

Olivier Georges, Pierre-Marie Gerlier, le cardinal militant 1880-1965, Paris, Desclée de Brouwer, 2014, $474 \mathrm{p}$. 
1 Si «le cardinal militant», choisi par Olivier Georges comme titre de sa biographie de Mgr Gerlier, n'a pas été expliqué dans une introduction de méthode, c'est que l'auteur n'entend pas imposer de présupposés. Le lecteur comme le chercheur doit donc juger sur pièces avec la documentation réunie et présentée. Le titre se comprend donc dans une quadruple filiation militante qui donne l'unité du personnage : le président de l'Association catholique de la jeunesse française (ACJF), le combattant de la Grande Guerre (miles christi rapidement prisonnier) devenu l'un des principaux chefs de l'Église militante issue de la Séparation et tournée vers l'Action catholique spécialisée.

2 Cette figure catholique, française et lyonnaise $\mathrm{du} \mathrm{xx}^{\mathrm{e}}$ siècle, certainement la plus brillante de la génération

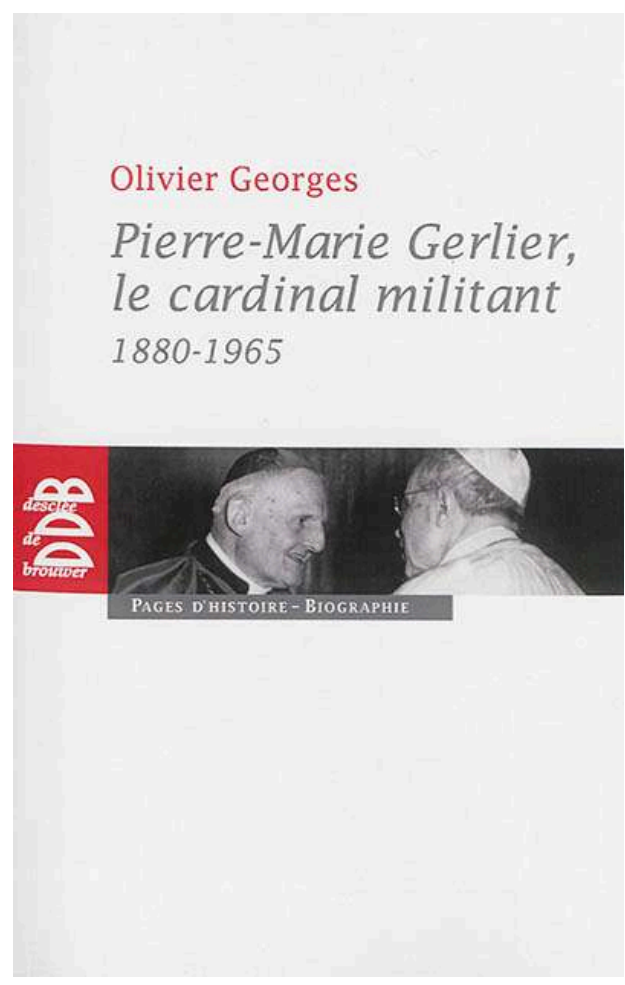
hiérarchique milieu de siècle, se prête évidemment à la biographie. On peut toutefois se demander si le genre convient bien pour saisir le foisonnement du milieu lyonnais au mitan $\mathrm{du} \mathrm{xx}^{\mathrm{e}}$ siècle, d'autant que les sources montrent en partie leurs limites en l'absence des fonds romains encore retenus après 1939. Mais il y a la richesse et l'intérêt des Archives lyonnaises (262 cartons !) qui font pâlir d'envie tout chercheur habitué des Archives diocésaines françaises, même s'il manque le journal personnel du primat des Gaules qui aurait permis d'entrer dans l'intimité du personnage. Les extraits spirituels cités ont beau être intéressants, ils participent de l'exercice codé de l'introspection.

3 Olivier Georges avance classiquement, par étapes chronologiques, en citant beaucoup. Cette dernière méthode aurait certainement gagné à n'être réservée qu'aux périodes « saillantes » (Vichy surtout, dont il faut détacher la description du voyage en Espagne de juin 1941 ; l'affaire de la condamnation des prêtres ouvriers), car pour les périodes « de transition » (années 1930 et 1950) cela tient parfois de l'énumération de l'agenda du pasteur.

Les titres de chapitre sont souvent percutants par leur simplicité (à l'image de « Temps nouveaux » pour qualifier la Libération qui précède « Le temps des affaires » de la fin $\mathrm{du}$ pontificat de Pie XII) et conduisent le développement, auquel il manque cependant un chapitre sur le «style Gerlier », dans l'esprit du « style épiscopal » qu'évoquait Yvon Tranvouez lors du colloque de Lyon de novembre 2010, et qui aurait donné vie et chair au tableau hiérarchique. De manière plus générale, la représentation de l'archevêque n'est pas toujours interrogée, et il en va de même pour la mémoire du personnage aux différentes échelles locales et nationales.

5 On soulignera donc le fait qu'Olivier Georges choisisse de traiter dans son chapitre 13 $\mathrm{du}$ «Juste parmi les Nations». L'identité et l'évaluation mémorielles servent significativement d'introduction à la recherche rigoureuse et chronologique pratiquée 
par l'auteur en particulier dans cette partie 1940-1944 de l'ouvrage. Ce choix n'est cependant pas sans interroger, car il subordonne ce qui a été la construction générale du maréchalisme à l'attitude hiérarchique face au drame juif. La mémoire, avec sa chronologie, méritait donc un chapitre en soi. Paradoxalement, c'est l'humour belge utilisé pour qualifier le voyage du cardinal à Bruxelles en janvier 1949 de «bénédiction à l'eau de Vichy» [note 66, p. 333] qui fait regretter cet oubli. Dans le même sens, Olivier Georges choisit la discrétion sur le long déclin du Prince de l'Église. Il ne s'agit pas bien sûr de critiquer l'élégance pudique de l'historien, mais de regretter que le biographe ne se soit pas saisi d'un portrait de la fin, notamment au concile. Ainsi l'émotion lyonnaise, comme celle qui a pu étreindre la ville à la mort d'Herriot, pouvait donner matière à une réflexion à ce qui fait l'image d'un archevêque dans la culture notamment populaire et encore municipale du début des années 1960. Le gaullisme finissant attribué au primat des Gaules méritait également d'être mieux expliqué car même si le soutien légaliste de Gerlier au moment du référendum de 1958 est incontestable, les relations personnelles n'ont jamais été excellentes comme le confirment les Mémoires de Peyrefitte.

Entre la soutenance de 2003 et la publication, le travail d'olivier Georges, bien que non mis à jour par des références à l'historiographie contemporaine, a surtout bénéficié de l'ouverture des archives de Pie XI. L'auteur donne ainsi les précisions attendues sur sa nomination épiscopale en 1929. Après avoir failli accéder à l'épiscopat dès 1927 (comme coadjuteur à Troyes et même auxiliaire de ce que j'ai pu voir quant à moi à Rome), il gagne donc le diocèse de Tarbes et Lourdes, siège que le cardinal Verdier trouve cependant un peu trop éloigné de Paris pour son ancien sous-directeur des œuvres. Évêque de Pie XI, il y impulse par le verbe et la présence une dynamisation certaine, même si l'auteur révèle à juste titre que l'Action catholique spécialisée a du mal à être lancée dans ce diocèse rural. Figure nationale et même européenne, Gerlier accède au prestigieux siège lyonnais en 1937. Il était pourtant loin d'être le premier candidat dans l'épiscopat français (Mgr Gaillard était pressenti) mais a bénéficié du soutien final de Pacelli et de Verdier dans une délicate sélection qu'éclaire bien l'auteur.

7 Pour conclure, ce travail rigoureux ne comporte que quelques rares erreurs, d'autant plus visibles (Suhard assistant à Vatican II, « Rhodain » au lieu de Rodhain, Mgr Martin évêque de Viviers pour le Puy). La lecture de ce Gerlier qui, tout en convainquant que la biographie d'un homme ne se réduit pas à une phase, ne fait pas changer d'avis: le discours du 19 novembre 1940, en donnant à l'ancien avocat une audience qu'il n'a jamais eue, rompt la trajectoire de toute une génération d'évêques. En citant in extenso cette allocution non improvisée, Olivier Georges ne signifie-t-il pas ainsi la fin d'un cycle biographique ou du moins de l'incroyable attrait pour ces discours maréchalistes qui captent les historiens depuis les années 1970 ? 